\title{
Extensão universitária e formação em saúde: experiências de um grupo tutorial do PET-Saúde Interprofissionalidade
}

\author{
Jamilly de Oliveira Musse² Érica Maria Granjeiro², Thais Moreira Peixoto3, Dyalle Costa e Silva4, Tamile \\ Rios Oliveira Almeida4, Tamires Barros de Carvalho4, Isabela Machado Sampaio Costa Soares³, Ivana
}

\section{Conceição Oliveira da Silva ${ }^{3}$}

\begin{abstract}
Resumo: A extensão universitária é um componente da formação que permite uma aprendizagem teórico-prática, maximizando o ensino obtido em sala de aula. O Programa de Educação pelo Trabalho para a Saúde/PET-Saúde Interprofissionalidade é um programa que objetiva o aperfeiçoamento e a especialização em serviço e estímulo à prática colaborativa. Este estudo relata as atividades desenvolvidas por um grupo tutorial do PET-Saúde Interprofissionalidade, da Universidade Estadual de Feira de Santana, no primeiro ano do programa. Trata-se de um relato de experiência, desenvolvido por estudantes (bolsistas de Farmácia, Medicina e Odontologia), dois professores (tutores, com formação em Odontologia e Fisioterapia) e cinco profissionais de saúde (preceptores, sendo 4 enfermeiras e 1 cirurgiã-dentista) no município de Feira de Santana - Bahia, tendo, como cenário de prática, o Centro de Atenção ao Diabético e Hipertenso. As ações foram desenvolvidas de abril a dezembro de 2019, e tiveram os resultados sistematizados em dois eixos: promoção da integração ensino-serviço-comunidade e desenvolvimento da docência e preceptoria em saúde. No eixo 1, desenvolveram-se oficinas de capacitação, reuniões, ações interprofissionais e de educação em saúde e elaboração de procedimentos operacionais padrão. No eixo 2, as atividades desenvolvidas permitiram a aproximação dos participantes com metodologias ativas de ensino, além de estimular a construção de artigos e apresentação de trabalhos em eventos. As ações realizadas contemplaram o diagnóstico situacional, atividades formativas e de educação em saúde, além do estímulo à produção científica, propiciando a reflexão dos participantes sobre a importância do cuidado interprofissional ao paciente diabético e hipertenso.
\end{abstract}

Palavras-chave: Educação Interprofissional; Universidade; Extensão Comunitária

\section{University extension and health education: tutorial group experiences in the Through Work Program in Interprofessional Health Education (PET-Health)}

\footnotetext{
Abstract: University extension is an educational component that allows theoretical-practical learning, maximizing classroom teaching. "Through Work Program in Interprofessional Health Education" (PET-Health) is a program that aims to improve and specialize in service and encourage collaborative practice. This study reports the activities of a PET-Health tutorial group from the Feira de Santana State University during the first year of the program. This article is an experience report developed by students (undergraduates in Pharmacy, Medicine and Dentistry), two teachers (tutors, bachelors in Dentistry and Physiotherapy), and five health professionals (tutors, four nurses, and one dentist) in the municipality of Feira de Santana (Bahia state, Brazil), with the Diabetic and Hypertensive Care Center as the practice scenario. The actions were carried out from April to December 2019, and the results were systematized in two axes: promotion of teaching-servicecommunity integration and development of teaching and health preceptorship. In axis 1, training workshops, meetings, interprofessional and health education actions, and standard operating procedures were developed. In axis 2, the activities developed allowed the participants to approach active teaching methodologies and encourage the construction of articles and presentation of papers at events. The performed actions included the situational diagnosis, training, health education activities, and encouragement of scientific production, providing the participants to reflect on the importance of interprofessional care to diabetic and hypertensive patients.
}

Keywords: Interprofessional Education; University; Community Outreach
Originais recebidos em 31 de julho de 2020

Aceito para publicação em 27 de março de 2021

1 Tutora do Projeto PETSaúde/Interprofissionalidade. Universidade Estadual de Feira de Santana (UEFS).

jamillymusse@gmail.com

(autora para correspondência)

2

Tutora do Projeto PET-

Saúde/Interprofissionalidade. Universidade Estadual de Feira de Santana (UEFS).

3 Preceptores do Projeto PETSaúde/Interprofissionalidade, Secretaria Municipal de Saúde de Feira de Santana, Bahia.

4 Bolsistas do Projeto PETSaúde/Interprofissionalidade, Universidade Estadual de Feira de Santana (UEFS). 


\section{Introdução}

A extensão é um componente da formação universitária que possibilita uma aprendizagem teórico-prática, maximizando o ensino obtido em sala de aula (Santos et al., 2016), além de promover a integração entre a academia e a sociedade, através da realização de atividades de assistência à população (Rodrigues et al., 2012). Trata-se de um processo que envolve interdisciplinaridade, cultura, educação, ciência e política, visando interagir e transformar tanto a universidade, quanto os outros setores sociais apoiados por ela. Neste contexto, as ações de extensão podem ser compreendidas também como práticas que unem o ensino e a pesquisa, promovendo assim, os valores democráticos, a equidade e o desenvolvimento social (Fórum de Pró-Reitores das Instituições Públicas de Educação Superior Brasileiras [FORPROEX], 2012).

De acordo com Santos et al. (2016), as práticas extensionistas promovem alterações consideráveis no âmbito estudantil e na sociedade, favorecendo o surgimento de novos métodos de pesquisa, novas percepções pela aproximação da teoria e prática, além da compreensão dos problemas sociais, econômicos e também políticos. Desta forma, torna possível o contato do estudante com a sociedade, trazendo benefícios para os dois grupos. Nesta perspectiva, de acordo com Rodrigues et al. (2012), o estudante apreende além do esperado, pois há uma satisfação maior ao se praticar os conhecimentos adquiridos em aula.

A Extensão Universitária é, portanto, uma das funções sociais da Universidade, que tem por objetivo promover o desenvolvimento social, fomentar projetos e programas de extensão que levam em conta os saberes e práticas populares, buscando garantir valores democráticos de igualdade de direitos, respeito à pessoa e sustentabilidade ambiental e social. Nesse sentido, uma das estratégias utilizadas pelo Ministério da Saúde foi a implementação do Programa de Educação pelo Trabalho para Saúde (PET-Saúde) Interprofissionalidade (Edital Ministério da Saúde/SGTES n 10, de 23 de julho de 2018), que tem como pressuposto o estímulo a educação interprofissional (EIP) e a prática colaborativa, configurando-se como ferramenta que busca qualificar o trabalho dos profissionais de saúde e aproximar os estudantes de graduação dos cursos de saúde, dos diversos cenários de práticas do Sistema Único de Saúde (SUS).

No que se refere à EIP, na literatura não há apenas uma definição sobre o termo, mas também a indicação de que não se trata apenas de juntar membros de diferentes profissões da saúde em um mesmo espaço (Souza \& Costa, 2019). A definição mais amplamente conhecida em todo o mundo é a do Centro para o Avanço da Educação Interprofissional (CAIPE), do Reino Unido, que a define como "a ocasião em que membros de duas ou mais profissões aprendem "com", "sobre" e "entre si" para melhorar a colaboração e a qualidade dos cuidados" (Barr \& Low, 2013).

Dessa forma, os autores ressaltam a importância das experiências de aprendizagem compartilhada, as quais permitem desenvolver a compreensão de que sempre é possível aprender com um membro de outra profissão, aprender uma ou outra forma de ver o mundo, um problema de saúde, uma abordagem ou conduta, com possibilidade de reorientação das práticas a partir desse encontro.

Para Câmara et al. (2016), a EIP necessita se estabelecer mediante o apoio institucional das universidades e se fortalecer com as políticas indutoras de formação, para tornar possível uma ampliação do diálogo com todas as profissões da saúde, bem como manter uma efetiva articulação entre os gestores dos serviços, em todos os níveis de governo, universidades, profissionais, órgãos reguladores das profissões, usuários, população, bem como escolas, a fim de superar os modelos predominantes de educação e prática uniprofissional, que não correspondem às reais necessidades de educação e saúde da população.

Ademais, de acordo com Almeida et al. (2019), a realidade de saúde da população, em sua complexidade, demonstra a necessidade do efetivo trabalho em equipe, com práticas colaborativas e ações integradas 
centradas no usuário, na família e na comunidade. Em se tratando do cuidado ao paciente diabético e hipertenso, a atuação interdisciplinar e interprofissional torna-se necessária para oferta de um cuidado adequado, visando atendimento integral dos indivíduos e familiares. Além disso, toda equipe deve estar capacitada para atuar na prevenção e controle, por meio de ações assistenciais, promoção e educação em saúde. Nesse contexto, a EIP é um instrumento relevante para transformar o processo formativo, visando a um avanço da colaboração, assim como da qualidade da atenção à saúde.

Diante disso, faz-se necessário o investimento na formação pedagógica e capacitação técnica dos profissionais de saúde, bem como o desenvolvimento de atividades educativas em saúde com usuário, familiares e comunidade, com o intuito de contemplar atividades de ensino, extensão e pesquisa. Assim, o presente trabalho teve como objetivo descrever as experiências do grupo tutorial PET-Saúde/Interprofissionalidade da Universidade Estadual de Feira de Santana (UEFS) atuante no Centro de Atenção ao Diabético e Hipertenso $(\mathrm{CADH})$, durante o primeiro ano de execução do programa.

\section{Metodologia}

Trata-se de um relato de experiência, de caráter descritivo, sobre as experiências de formação e extensão realizadas pelo grupo tutorial 4 do PET-Saúde/Interprofissionalidade UEFS, atuantes no CADH, Feira de Santana, Bahia, durante o primeiro ano do Programa, entre abril de 2019 e abril de 2020. O CADH é um centro especializado de gestão municipal, considerado uma unidade de média complexidade, fundada no ano de 2001, como estratégia de fortalecimento da linha do cuidado e tendo como finalidade assistir aos usuários diabéticos de alto risco cardiovascular, cuja complexidade não poderá ser resolvida apenas na Atenção Primária (Nunes et al., 2020).

O grupo tutorial é formado por seis estudantes regularmente matriculados nos cursos de Farmácia (dois), Medicina (dois) e Odontologia (dois) da UEFS, cinco preceptoras com graduação em enfermagem e odontologia, que atuam na Secretaria Municipal de Saúde do município, e duas professoras (tutoras) do curso de Medicina da UEFS, com bacharelado em Odontologia e Fisioterapia, totalizando 13 integrantes. No Quadro 1 está representada a composição da equipe interprofissional do Pet/Saúde/Interprofissionalidade da UEFS. A composição interdisciplinar tem por intuito expandir a discussão da mudança curricular nucleada em cada curso, sem dispensar as interfaces e a integração dos processos de mudança dos cursos.

Quadro 1. Equipe interprofissional do Programa Educação pelo Trabalho, PET-Saúde Interprofissionalidade da Universidade Estadual de Feira de Santana (UEFS), 2019-2021.

\begin{tabular}{|c|c|c|c|c|c|c|}
\hline \multicolumn{7}{|c|}{ Equipe interprofissional PET- Saúde UEFS (grupo 04) } \\
\hline \multicolumn{5}{|c|}{ UEFS/ Cursos Nível Superior/ número de participantes } & \multirow{2}{*}{\multicolumn{2}{|c|}{$\begin{array}{c}\text { CADH/SMS } \\
\begin{array}{l}\text { Profissionais de Saúde/ } \\
\text { Preceptoras }\end{array} \\
\text { Formação/Bacharelado }\end{array}$}} \\
\hline \multicolumn{2}{|c|}{$\begin{array}{c}\text { Docentes/Tutoras } \\
\text { Formação/Bacharelado }\end{array}$} & \multicolumn{3}{|c|}{$\begin{array}{l}\text { Bacharelado/ Discentes/Cursos de } \\
\text { Graduação }\end{array}$} & & \\
\hline Odontologia & Fisioterapia & Farmácia & Medicina & Odontologia & Odontologia & Enfermagem \\
\hline 1 & 1 & 2 & 2 & 2 & 1 & 4 \\
\hline
\end{tabular}


Todas as atividades foram desenvolvidas em caráter participativo, com anuência dos profissionais de saúde do cenário de prática, e tendo como base as diretrizes da educação interprofissional, sendo supervisionadas pelas tutoras.

Foram priorizados nesse trabalho dois eixos temáticos propostos pelo PET-Saúde/Interprofissionalidade, sendo denominados de: Eixo 1- Promoção da Integração ensino-serviço-comunidade e Eixo 2Desenvolvimento da docência e da preceptoria na saúde.

Para cada eixo foram descritas as atividades específicas realizadas pelo grupo tutorial, de forma a atender aos objetivos propostos para o primeiro ano do programa: qualificar a docência e a preceptoria na saúde para utilização dos fundamentos teóricos-conceituais e metodológicos da EIP; desenvolver e ampliar ações compartilhadas de educação e trabalho interprofissional em saúde.

As ações foram realizadas em diferentes setores e cenários (Universidades, CADH, Ginásio Municipal, Secretaria Municipal de Saúde de Feira de Santana - Bahia, dentre outros). As atividades foram organizadas e priorizadas, respeitando o horário e grade curricular dos estudantes, bem como os turnos de trabalhos dos profissionais de saúde dos cenários envolvidos.

\section{Resultados}

As ações realizadas contemplaram os dois eixos temáticos do programa e foram representadas no Quadro 2 com a descrição das experiências pedagógicas de cada atividade desenvolvida pelo grupo, seus objetivos, carga-horária, público participante e local de realização.

\section{Eixo 1- Promoção da Integração ensino-serviço-comunidade}

No eixo temático 1 , que trata sobre a descrição das atividades que promovem a integração ensino-serviçocomunidade, com foco no desenvolvimento do SUS, elaborou-se inicialmente o diagnóstico situacional do cenário de prática, permitindo ao grupo tutorial acompanhar e conhecer a rotina de usuários diabéticos e hipertensos cadastrados no serviço especializado; avaliar a percepção dos mesmos sobre o processo saúdedoença e compreender o funcionamento do serviço, com vistas a levantar a demanda de necessidades de saúde e possíveis temáticas a serem trabalhadas nas atividades de educação em saúde, com o público participante e capacitações teórico-práticas com a equipe de saúde do serviço. A importância dessa ação inicial possibilitou ao grupo um conhecimento prévio sobre o cenário de atuação, bem como a publicação dessa experiência em periódico nacional (Nunes et al., 2020).

Outras ações organizadas pelo grupo envolveram a realização de um seminário intitulado: "Atual cenário do cuidado multiprofissional em Diabetes" e um simpósio intitulado "Simpósio de Atenção Interprofissional em Saúde", tendo como público os profissionais de saúde, e como objetivo refletir sobre o cuidado multiprofissional ao paciente diabético. Realizou-se também uma capacitação teórico-prática para enfermeiros do município, com objetivo de orientá-los quanto ao uso correto das canetas de glicemia, além de uma atividade comemorativa ao dia Mundial do Diabetes em novembro de 2019 (Figura 1A-E). Essa ação em comemoração ao Dia Mundial do Diabetes é realizada anualmente pela equipe do $C A D H$, tendo como público participante os usuários cadastrados no centro, familiares e a comunidade em geral. O grupo tutorial foi parceiro na organização do evento, participando de reuniões de planejamento, confecção de cartaz, folder e cartão para registro dos participantes nas atividades disponibilizadas. Durante o evento, os bolsistas, preceptores e tutores participaram das ações voltadas para orientação em saúde da clientela, monitoramento da pressão arterial, medição do peso, altura, glicemia casual, cálculo do Índice de Massa Corpórea (IMC), além da participação na triagem de pacientes adultos para rastreamento da Diabetes Mellitus e possíveis 
encaminhamentos para as unidades de saúde, sob orientação dos profissionais do CADH. A ação contou também com a participação de oferta de serviços de nutrição, endocrinologia, orientação de higiene bucal, exame odontológico, práticas terapêuticas não convencionais, dentre outros, em parceria com outras instituições de saúde.

Quadro 2. Atividades realizadas pelo Grupo Tutorial 4 do PET-Saúde/Interprofissionalidade UEFS durante $\circ 1^{\circ}$ ano de atividades, 2019-2020.

\begin{tabular}{|c|c|c|c|c|c|}
\hline EIXO TEMATICO & $\begin{array}{l}\text { EXPERIÊNCIA } \\
\text { PEDAGÓGICA }\end{array}$ & OBJETIVO & PÚBLICO-ALVO & $\begin{array}{l}\text { CARGA } \\
\text { HORÁRIA }\end{array}$ & LOCAL \\
\hline \multirow{6}{*}{$\begin{array}{l}\text { Promoção da } \\
\text { integração ensino- } \\
\text { serviço-comunidade }\end{array}$} & $\begin{array}{l}\text { Diagnóstico } \\
\text { situacional (citar o } \\
\text { artigo nas } \\
\text { referências) }\end{array}$ & $\begin{array}{l}\text { Conhecer o cenário de } \\
\text { prática }\end{array}$ & Grupo 04 PET & $64 \mathrm{~h}$ & $\mathrm{CADH}^{1}$ \\
\hline & $\begin{array}{l}\text { Seminário "Atual } \\
\text { cenário do cuidado } \\
\text { multiprofissional em } \\
\text { Diabetes" }\end{array}$ & $\begin{array}{l}\text { Promover a educação } \\
\text { permanente dos } \\
\text { profissionais de saúde }\end{array}$ & $\begin{array}{l}\text { Profissionais de } \\
\text { saúde do } \\
\text { município }\end{array}$ & $4 h$ & $\begin{array}{l}\text { Auditório } \\
\mathrm{SMS}^{2}\end{array}$ \\
\hline & $\begin{array}{l}\text { I Simpósio de } \\
\text { Atenção } \\
\text { Interprofissional em } \\
\text { saúde: um olhar } \\
\text { sobre o paciente } \\
\text { diabético }\end{array}$ & $\begin{array}{l}\text { Discutir o cuidado } \\
\text { interprofissional ao } \\
\text { paciente diabético }\end{array}$ & $\begin{array}{l}\text { Enfermeiros, } \\
\text { cirurgiões- } \\
\text { dentistas e } \\
\text { nutricionistas da } \\
\text { Atenção } \\
\text { Primária }\end{array}$ & $8 \mathrm{~h}$ & $\begin{array}{l}\text { Auditório } \\
\text { SMS }\end{array}$ \\
\hline & $\begin{array}{l}\text { Oficina de } \\
\text { capacitação do uso } \\
\text { da caneta glicemia }\end{array}$ & $\begin{array}{l}\text { Orientar os enfermeiros } \\
\text { da Atenção Primária no } \\
\text { uso das canetas de } \\
\text { glicemia }\end{array}$ & $\begin{array}{l}\text { Enfermeiros da } \\
\text { Atenção } \\
\text { Primária }\end{array}$ & $4 \mathrm{~h}$ & $\begin{array}{l}\text { Auditório } \\
\text { SMS }\end{array}$ \\
\hline & $\begin{array}{l}\text { Dia Mundial do } \\
\text { Diabetes }\end{array}$ & $\begin{array}{l}\text { Integrar profissionais, } \\
\text { usuários e familiares } \\
\text { em atividades } \\
\text { educativas, } \\
\text { assistenciais e } \\
\text { momentos de lazer. }\end{array}$ & $\begin{array}{l}\text { Familiares e } \\
\text { usuários } \\
\text { diabéticos do } \\
\text { município }\end{array}$ & $6 \mathrm{~h}$ & $\begin{array}{l}\text { Ginásio } \\
\text { de } \\
\text { Esportes } \\
\text { Joselito } \\
\text { Amorim }\end{array}$ \\
\hline & $\begin{array}{l}\text { Implementação dos } \\
\text { POP }^{3} \text { pra unidade }\end{array}$ & $\begin{array}{l}\text { Padronização das } \\
\text { rotinas do serviço }\end{array}$ & $\begin{array}{l}\text { Profissionais do } \\
\text { CADH }\end{array}$ & $32 \mathrm{~h}$ & $\mathrm{CADH}$ \\
\hline \multirow{3}{*}{$\begin{array}{l}\text { Desenvolvimento } \\
\text { da docência e da } \\
\text { preceptoria na } \\
\text { saúde }\end{array}$} & $\begin{array}{l}\text { Apresentação de } \\
\text { trabalhos científicos } \\
\text { em eventos }\end{array}$ & $\begin{array}{l}\text { Divulgar o programa e } \\
\text { atividades do grupo }\end{array}$ & $\begin{array}{l}\text { Preceptora do } \\
\text { grupo PET }\end{array}$ & $2 \mathrm{~h}$ & UNEF $^{4}$ \\
\hline & $\begin{array}{l}\text { Oficina de } \\
\text { construção dos POP }\end{array}$ & $\begin{array}{l}\text { Instrumentalizar a } \\
\text { equipe tutorial na } \\
\text { elaboração e discussão } \\
\text { dos POP de } \\
\text { Enfermagem }\end{array}$ & Grupo PET & $4 h$ & $\begin{array}{l}\text { Sala da } \\
\text { VIEP }^{5}\end{array}$ \\
\hline & $\begin{array}{l}\text { Metodologias ativas: } \\
\text { estudo de caso }\end{array}$ & $\begin{array}{l}\text { Discutir a atenção } \\
\text { interprofissional no } \\
\text { cuidado ao paciente } \\
\text { diabético }\end{array}$ & $\begin{array}{l}\text { Grupo PET, } \\
\text { enfermeiros, } \\
\text { nutricionistas e } \\
\text { cirurgiões- } \\
\text { dentistas da } \\
\text { Atenção } \\
\text { Primária }\end{array}$ & $8 \mathrm{~h}$ & $\begin{array}{l}\text { Auditório } \\
\text { SMS }\end{array}$ \\
\hline
\end{tabular}

Notas:

1 CADH: Centro de Atendimento ao Diabético e Hipertenso; 2 SMS: Secretaria Municipal de Saúde; 3 POP: Procedimento Operacional Padrão; 4 UNEF: Unidade de Ensino Superior de Feira de Santana; 5 VIEP: Vigilância Epidemiológica 


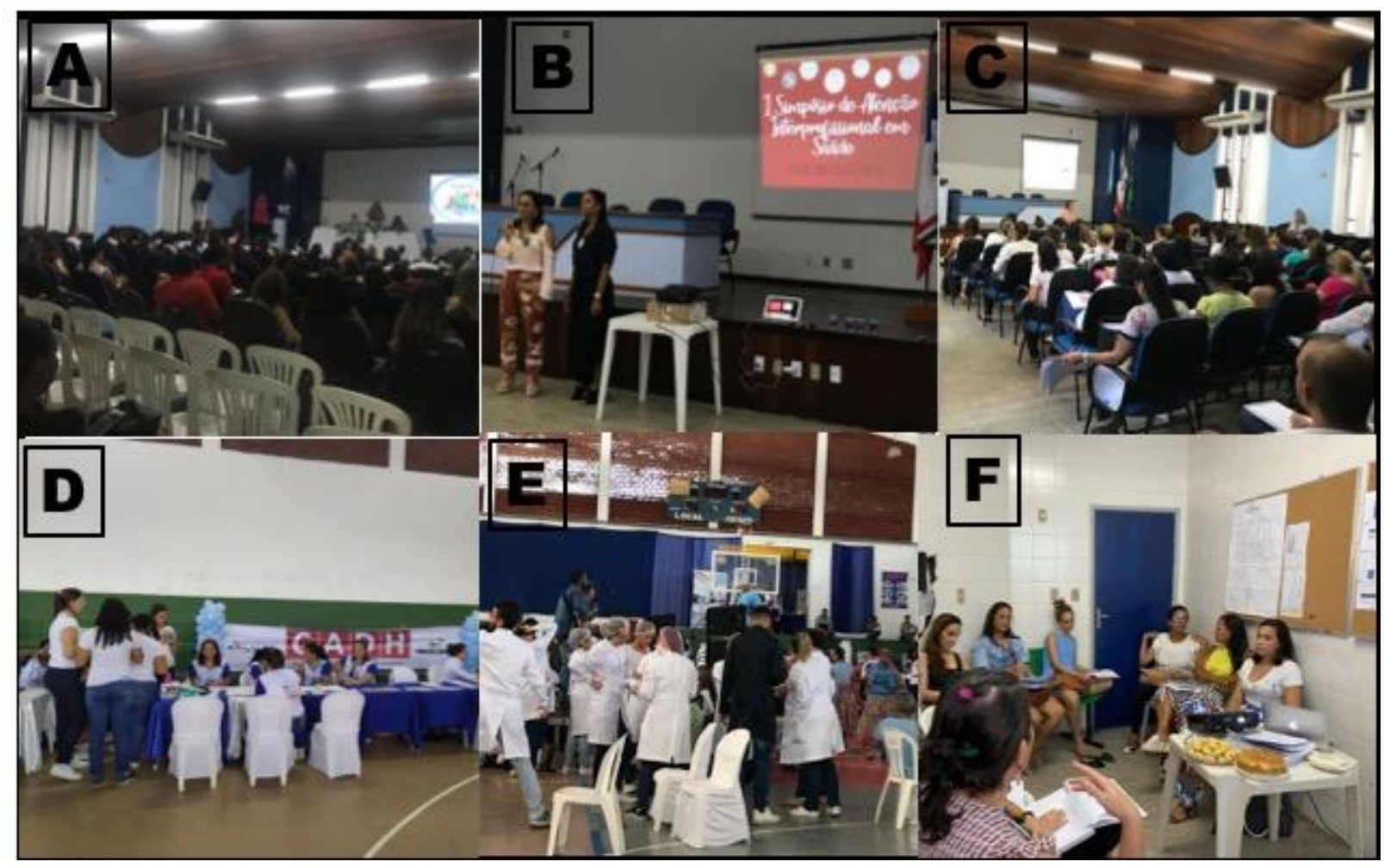

Figura 1. Imagens ilustrativas das experiências pedagógicas vivenciadas pela equipe PET Saúde Interprofissionalidade, atuante no Centro de Atenção ao Diabético e Hipertenso (CADH). A. Seminário. B. I Simpósio de Atenção Interprofissional em Saúde. C. Oficina de capacitação para utilização da caneta de medidas glicêmicas. D-E. Ação comunitária durante o Dia Mundial do Diabetes do ano de 2019. F. Oficina com o grupo tutorial de construção dos POP.

A atuação do grupo tutorial durante o primeiro ano de atividades no $C A D H$ despertou nos integrantes, a partir do diagnóstico situacional e conhecimento do processo de trabalho da equipe de saúde, a necessidade de padronizar alguns protocolos e práticas dos profissionais. Assim, a partir do diálogo com os profissionais e coordenação do serviço foram listados pelos integrantes as rotinas que necessitam ser padronizadas.

\section{Eixo 2- Desenvolvimento da docência e da preceptoria na saúde}

No eixo temático 2 foi abordado o desenvolvimento da docência e da preceptoria na saúde, tendo como objetivo qualificar a docência e a preceptoria na saúde para utilização dos fundamentos teóricos e metodológicos da EIP e ampliar ações compartilhadas de educação e trabalho interprofissional em saúde. Assim, as atividades desenvolvidas instrumentalizaram preceptores e tutores para a realização de ações de educação e trabalho interprofissional, com participação e organização de oficinas para construção de procedimentos operacionais padrão (POP) (Figura 1- F), utilizando metodologias ativas; discussão de casos clínicos e artigos científicos, além de apresentação de trabalhos em congressos na forma de relatos de experiência, com objetivo de divulgar o programa e as atividades do grupo.

A construção dos POP partiu do relato e observação dos profissionais no desenvolvimento da assistência ao usuário, onde o grupo tutorial foi desenhando os fluxos e procedimentos para cada atividade vivenciada, com 
vistas à construção do protocolo, considerado uma ferramenta gerencial do profissional para padronizar os processos de trabalho da equipe, reduzindo as chances de erros na execução dos procedimentos. A elaboração dos documentos pelo grupo contou com a condução das enfermeiras do serviço, considerando que alguns destes docuemntos são específicos para descrição das ações da Enfermagem, e porque o grupo tutorial não possuía integrantes do curso de enfermagem. Os protocolos foram apreciados pela equipe de saúde e coordenação da unidade, e posteriormente disponibilizados à instituição, como contribuição para melhoria da assistência.

Os POP elaborados nessa ação incluíram: POP de lavagem das mãos; POP de higienização com álcool a 70\%; POP de consulta de Enfermagem para monitoramento e dispensação da fita de glicemia; POP de consulta de Enfermagem para triagem/acolhimento de novos usuários e POP de Enfermagem à Pessoa com Diabetes Mellitus e/ou Hipertensão.

A apresentação dos primeiros trabalhos do grupo em eventos aconteceu no mês de novembro de 2019, durante o II Congresso de Odontologia da Unidade de Ensino Superior de Feira de Santana (II COUNEF), realizado na sede da própria instituição, no município de Feira de Santana - Bahia. Neste evento, dois trabalhos foram aprovados, um na categoria tema livre e outro como painel, sendo eles: "Diagnóstico situacional no planejamento estratégico em saúde: relato de experiência da inserção da Odontologia no PET-Saúde Interprofissionalidade" e "Educação interprofissional e metodologias ativas: importância na formação do acadêmico de Odontologia", respectivamente.

Por fim, buscando estimular o desenvolvimento de atividades que contemplem metodologias ativas no processo de ensino-aprendizagem, foi realizada uma oficina com profissionais da atenção primária (enfermeiros, nutricionistas e cirurgiões-dentistas), com discussão de caso clínico envolvendo o cuidado ao paciente diabético e o estímulo à prática interprofissional.

\section{Discussão}

O PET-Saúde/Interprofissionalidade aplica as bases teóricas e metodológicas da EIP - método educacional que tem como finalidade formar profissionais mais aptos para o trabalho colaborativo em saúde, permitindo uma experiência favorável, uma vez que o envolvimento serviço-ensino-comunidade possibilita uma interação entre os diversos setores e uma aproximação precoce dos estudantes com o SUS.

Nesse sentido, as ações inseridas no eixo 1 do programa, executadas pelo grupo tutorial PETSaúde/Interprofissionalidade UEFS, tanto no cenário de prática (diagnóstico situacional, elaboração dos POP), quanto com profissionais de saúde (seminários, simpósio e oficinas), pacientes e familiares (atividade comemorativa ao dia mundial do diabetes e acompanhamento da rotina destes no serviço) permitiram aos bolsistas, preceptores e tutores conhecer alguns dos determinantes e condicionantes sociais do processo saúde-doença, dialogar com os diversos atores sociais envolvidos nesse processo, incentivando a reflexão sobre a importância da atuação interprofissional no cuidado ao paciente diabético, além de contribuir com a implementação de mudanças para melhorias da rotina do serviço.

No que se refere à formação dos profissionais de saúde, considerando a complexidade do processo saúdedoença-cuidado, Guimarães (2017) aponta para a necessidade da implementação de um conjunto de iniciativas, na perspectiva de superar as práticas tradicionais de ensino, articulando o mundo da formação com o do trabalho, com vistas ao desenvolvimento de um perfil profissional generalista, crítico, reflexivo, humanista e capaz de intervir nos principais problemas de saúde da população. Dentro dessa perspectiva, no presente trabalho, foram realizadas algumas oficinas que buscaram refletir sobre os desafios do cuidado 
interprofissional ao paciente diabético, com uma abordagem inicialmente teórica, seguida pela discussão de casos, com os participantes divididos em grupos com formação multiprofissional.

Para Paviani e Fontana (2009) e Granjeiro et al. (2020), a aplicação de oficinas no ensino é uma estratégia potencial para a aprendizagem, sendo um processo ativo de transformação recíproca entre sujeito e objeto. Sendo assim, o uso de oficinas pode auxiliar no processo de ensino aprendizagem, pois representa uma ferramenta metodológica muitas vezes capaz de tornar o ensino em saúde atraente, além de facilitar a compreensão de seus conteúdos.

Entre os eventos educativos realizados pelo grupo, destaca-se uma atividade comemorativa ao Dia Mundial da Diabetes. Segundo a Diretriz da Sociedade Brasileira de Diabetes (2019), a Diabetes Mellitus é um distúrbio metabólico caracterizado por hiperglicemia persistente que está associada a complicações crônicas micro e macrovasculares, acarretando elevação da morbidade e redução da qualidade de vida. Visando alcançar a orientação e rastreamento da comunidade local, o grupo do PET, juntamente com a equipe do CADH, realizou atividades de aferição da pressão, peso, glicemia e cálculo do IMC, avaliação nutricional, consulta com endocrinologista e avaliação odontológica, além orientação sobre a importância da prática de atividade física.

A Organização Pan-Americana da Saúde (OPAS) entende que a Educação em Saúde deve focar em uma perspectiva integral, na criação de ambientes e entorno saudáveis, e em uma articulação com os equipamentos sociais do território, como por exemplo, as unidades e serviços de saúde.

De acordo com Guimarães (2017), deve-se promover saúde a partir do diálogo, da reflexão sobre as realidades, dos valores, das individualidades dos sujeitos e de ações coletivas. Para Salci et al. (2013), a educação em saúde é compreendida como um processo complexo, pois envolve dimensões políticas, filosófica, social, religiosa além de envolver aspectos práticos e teóricos do indivíduo, grupo, comunidade e sociedade. Assim, envolve o processo saúde-doença para evitar e/ou retardar a presença de doença, resgate da qualidade de vida, evitando complicações do processo de adoecimento.

Diante do exposto, percebe-se, que as práticas educativas voltadas para os pacientes com Diabetes Mellitus constituem uma ferramenta que estimula a participação ativa da população em diversas esferas, como no planejamento, desenvolvimento e implantação das atividades educativas. Logo, maximiza o conhecimento dos alunos, tutores e preceptores, proporcionando maior aproximação ao contexto social da população, e refletindo sobre as potencialidades e desafios da atuação interprofissional.

Outro ponto importante das ações desenvolvidas pelo PET foi a inserção na rotina do cenário de prática, o $C A D H$, permitindo a integração com os profissionais, usuários do serviço e familiares, e a realização de atividades de educação em saúde, voltadas para o público do serviço. A experiência vivenciada pelo grupo tutorial no cenário do $\mathrm{CADH}$, com diagnóstico situacional, possibilitou o planejamento de abordagens direcionadas às ações de promoção e prevenção, buscando melhorias na qualidade de vida de usuários cadastrados (Nunes et al., 2020). Além disso, apontou algumas fragilidades do serviço, dentre elas a necessidade de padronização de algumas rotinas por parte dos profissionais.

Em função disso, deu-se início às capacitações internas do grupo (oficina mencionada no segundo eixo do quadro 2) e a construção do procedimento operacional padrão (POP) de cada setor que compõe o referido centro, culminando, neste primeiro ano, na confecção dos POP vinculados aos setores de enfermagem. Segundo Toso et al. (2015), o POP deve ser entendido como uma sistematização dos processos, que permite a equipe ordenar a execução de determinado procedimento, viabilizando que estes sejam seguros e embasados em evidencias científicas, o que confere maior confiabilidade à assistência.

A oficina de construção dos POP foi conduzida por uma das preceptoras do grupo tutorial, com formação em enfermagem. Também contou com a participação de uma convidada externa ao grupo, também enfermeira, 
que na ocasião estava como estudante em prática de residência Multiprofissional em Saúde da Família no CADH. A experiência contribuiu para consolidar o desenvolvimento de ações inseridas no segundo eixo do programa, visando estimular a docência e preceptoria em saúde.

Além disso, tutores e preceptores foram estimulados à produção científica, através da redação de artigos e apresentação de trabalhos em congressos, e tiveram a oportunidade de experimentar contextos e práticas pedagógicas diferentes, com o desenvolvimento e participação direta em atividades envolvendo discussão de casos clínicos, utilizando metodologias ativas de ensino. Para Borges e Alencar (2014) o uso de metodologias ativas estimula a curiosidade e participação dos indivíduos, tornando-os protagonistas do processo de ensinoaprendizagem. Nesse sentido, a oficina realizada pelo grupo, juntamente com profissionais de saúde do município, descrita no Quadro 2, eixo 2 do programa, que trabalhou com a aprendizagem baseada em problemas, através da discussão de um caso clínico, contribuiu para reflexão dos participantes sobre a importância da prática colaborativa no cuidado ao paciente diabético. Apesar de o grupo participante da referida oficina reconhecer a importância de uma atuação interprofissional no cuidado aos pacientes, percebeu-se, na prática, acompanhando o serviço, uma atuação multidisciplinar, com especialidades e procedimentos isolados. Percepção semelhante também foi apontada por Zingra et al. (2020).

Entre os fatores limitantes para a inserção da EIP, definidos por Almeida et al. (2019), encontra-se o apoio institucional, quando este não conhece os efeitos positivos para a plenitude do cuidado e não valoriza a prática interprofissional. Neste aspecto o apoio institucional da UEFS e da gestão do CADH foram imprescindíveis para realização das atividades do grupo tutorial, contribuindo para consolidação da interação entre ensino, serviço e comunidade, e fortalecendo a docência e preceptoria em saúde.

\section{Conclusão}

O PET-Saúde/ Interprofissionalidade contribuiu para o fortalecimento de uma prática acadêmica integrada entre serviço de saúde e usuários, atendendo as demandas do cenário de prática. As ações desenvolvidas pelo grupo tutorial foram bastante diversificadas, envolvendo o conhecimento sobre o território de atuação através da realização do diagnóstico situacional, atividades formativas e de educação em saúde, além da elaboração e publicação de artigos científicos, procedimentos operacionais padrão (POP) e apresentação de trabalhos acadêmicos. Estas atividades possibilitaram a reflexão dos participantes sobre a importância do cuidado interprofissional ao paciente diabético e hipertenso, impactando de forma positiva no cenário de prática e estilo de vida do usuário, fortalecendo o vínculo da equipe de saúde e do PET com os mesmos, através das ações educativas, e favorecendo a articulação de atividades de pesquisa, ensino e extensão universitária. Tornase essencial para a formação dos estudantes o reconhecimento das especificidades do território de atuação, com atividades que promovam o desenvolvimento de habilidades para o trabalho interprofissional, bem como o envolvimento das diferentes profissões nas discussões do cotidiano de trabalho em saúde.

\section{Contribuições de cada autor}

Todos os autores participaram da redação do artigo e todas as etapas descritas na metodologia.

\section{Referências}

Almeida, R. G. S., Teston, E. F., \& Medeiros, A. A. (2019). A interface entre o PET-Saúde/Interprofissionalidade e a Política Nacional de Educação Permanente em Saúde. Saúde Debate, 43(1): 97-105.

Barr, H., \& Low, H. (2013). Introdução à Educação Interprofissional. Fareham: CAIPE. 
Borges, T. \& Silva, A. G. (2014). Metodologias ativas na promoção da formação crítica do estudante: 0 uso das metodologias ativas como recurso didático na formação crítica do estudante do ensino superior. Cairu em Revista, 4(3): 119-143.

Câmara, A. M. C. S., Cyrino, A. P., Cyrino, E. G., Azevedo, G. D., Costa, M. V. D., Bellini, M. I. B., ... \& Reeves, S. (2016). Educação interprofissional no Brasil: Construindo redes formativas de educação e trabalho em saúde. Interface, 20(56): 5-8.

Fórum de Pró-Reitores das Instituições Públicas de Educação Superior Brasileiras (2012). Política Nacional de Extensão Universitária. Manaus: FORPROEX. Recuperado de: https://proex.ufsc.br/files/2016/04/Pol\%C3\%ADtica-Nacional-deExtens\%C3\%A3o-Universit\%C3\%A1ria-e-book.pdf

Granjeiro, É. M., Musse, J. O., Peixoto, T. M., Nunes, I. V., Soares, I. M. S. C., Silva, I. C. O., ... \& Dias, Y. O. (2020). Estratégias de ensino à distância para a educação interprofissional em Saúde frente à pandemia COVID-19. Revista de Divulgação Científica Sena Aires, 9: 591-602.

Guimarães, E. M. S. (2017). Expressões conservadoras no trabalho em saúde: A abordagem familiar e comunitária em questão. Serviço Social \& Sociedade, 130: 564-582.

Nunes, I. V., Santos, R. C., Dias, Y. O., Peixoto, T. M., Pereira, E. C. S., Silva, A. S. J., ... \& Musse, J. O. (2020). Acompanhamento de pacientes adultos com diabetes e hipertensão em Centro Especializado: A experiência do PETSaúde Interprofissionalidade. Revista de Divulgação Científica Sena Aires, 9(1): 304-12.

Paviani, N. M. S. \& Fontana, N. M. (2009). Oficinas pedagógicas: Relato de uma experiência. Conjectura, 14(2): 77-88.

Rodrigues, A. Á. A. D. O., Juliano, I. A., Melo, M. L. C., Beck, C. L. C., \& Prestes, F. C. (2012). Processo de interação ensino, serviço e comunidade: a experiência de um PET-Saúde. Revista Brasileira de Educação Médica, 36(2), 184-192.

Salci, M. A., Maceno, P., Rozza, S. G., Silva, D. M. G. V., Boehs, A. E. \& Heidemann, I. T. S. B. (2013). Educação em Saúde e suas perspectivas teóricas: Algumas reflexões. Revista Texto \& Contexto - Enfermagem, 1(1): 224-230.

Santos, J. H. S., Rocha, B. F. \& Passaglio, K. T. (2016). Extensão Universitária e Formação no Ensino Superior. Revista Brasileira de Extensão Universitária, 7(1): 23-28.

Sociedade Brasileira de Diabetes (2019). Diretriz da sociedade brasileira de diabetes 2019-2020. Recuperado de: https://www.diabetes.org.br/profissionais/images/DIRETRIZES-COMPLETA-2019-2020.pdf

Souza, R. M. P. \& Costa, P. P. (2019). Nova formação em saúde pública: Aprendizado coletivo e lições compartilhadas na RedEscola. Rio de Janeiro: Fiocruz.

Toso, B. R. G. O., Viera, C. S., Valter, J. M., Delatore, S. \& Barreto, G. M. S. (2015). Validação de protocolo de posicionamento de recém-nascido em Unidade de Terapia Intensiva. Revista Brasileira de Enfermagem, 68(6): 11471153.

Zingra, K. N. S., Silva, A. C. R., Silva, Í. D. G., Torres, A. S. L. \& Branco Júnior, A. G. (2020). Utilização do método case based learning em uma oficina de educação interprofissional para profissionais docentes da área de saúde: Relato de experiência. Diálogos, 1: 18-24.

Como citar este artigo:

Musse, J. de O., Granjeiro, E. M., Peixoto, T. M., Costa e Silva, D., Almeida, T. R. O., de Carvalho, T. B., Soares, I. M. S. C., \& da Silva, I. C. O. (2021). Extensão universitária e formação em saúde: Experiências de um grupo tutorial do PET-Saúde Interprofissionalidade. Revista Brasileira de Extensão Universitária, 12(1), 103-112. https://periodicos.uffs.edu.br/ index.php/RBEU/article/view/11637/pdf 